\title{
REMARK IN REGARD TO BIDELMAN'S DATA-BANK PROJECT
}

\author{
W. P. BIDELMAN \\ Warner and Swasey Observatory, Case Western Reserve University, Cleveland, Ohio, U.S.A.
}

(Read by C. B. Stephenson)

The concept of a general stellar data bank is not a new idea, but the desirability of this project at the present time has been emphasized by a number of astronomers (Bidelman, Jaschek, Cayrel, Hauck and others) and work is proceeding both in the United States and elsewhere. The status of my own activities in this direction is as follows:

The general stellar data file now at the Warner and Swasey Observatory is continually being updated and much more of the astronomical literature is being systematically searched and the resulting data and references entered into the file. However this project is still largely unfunded and progress is far slower than desirable. In particular the file is still not available in machine-readable form, though several of the newer large data catalogues, such as the Warner and Swasey southern OB-star survey of Stephenson and Sanduleak, will be entered into the file in IBM card-form rather the entire file will be converted to punched cards.

Efforts during the past year to obtain increased funds for this project from the

Efforts during the past year to obtain increased funols for this project from the astronomy program of the NSF have proved of little avail, but it is intended to submit a proposal to the Scientific Information Office of the NSF, which is more particularly concerned with such a project, to support hiring one or more persons whose entire activity would be concerned with the data file. This should make it possible to bring the file essentially up to date within a year or two - at least for references of astrophysical interest - and to make a good start on converting the present file into a more useful one.

If financial support for this project is to be obtained from Federal sources it is clear that the work must have the enthusiastic support of the entire astronomical community; further, the agencies involved would like to be assured that the work will not be needlessly duplicated elsewhere and that the data will indeed be used. Expressions of support for our work will be much appreciated. 